Meta

Journal des traducteurs

Translators' Journal

\title{
La traduction des voix françaises en igbo : problèmes et solutions
}

\section{Benedict O. Okiwelu}

Volume 44, numéro 4, décembre 1999

URI : https://id.erudit.org/iderudit/003761ar

DOI : https://doi.org/10.7202/003761ar

Aller au sommaire du numéro

Éditeur(s)

Les Presses de l'Université de Montréal

ISSN

0026-0452 (imprimé)

1492-1421 (numérique)

Découvrir la revue

Citer cet article

Okiwelu, B. O. (1999). La traduction des voix françaises en igbo : problèmes et solutions. Meta, 44(4), 650-660. https://doi.org/10.7202/003761ar
Résumé de l'article

Dans les départements des langues modernes européennes des universités nigérianes, les cours de traduction font partie intégrante du programme d'études mais sont limités à l'orientation français-anglais-français ou bien allemand-anglais-allemand. La traduction pratique du français-langue maternelle-français ne se conçoit guère à cause de notre tradition éducative coloniale. Ce travail aura ainsi pour tâche de traduire les voix françaises en igbo, une des trois principales langues du Nigeria, tout en soulevant les problèmes de traduction qui se posent et en proposant les solutions qui s'imposent. 


\title{
BLOC-NOTES
}

\author{
La traduction des voix françaises en igbo: problèmes et solutions
}

\begin{abstract}
RÉSUMÉ
Dans les départements des langues modernes européennes des universités nigérianes, les cours de traduction font partie intégrante du programme d'études mais sont limités à l'orientation français-anglais-français ou bien allemand-anglais-allemand. La traduction pratique $d u$ français-langue maternelle-français ne se conçoit guère à cause de notre tradition éducative coloniale. Ce travail aura ainsi pour tâche de traduire les voix françaises en igbo, une des trois principales langues du Nigeria, tout en soulevant les problèmes de traduction qui se posent et en proposant les solutions qui s'imposent.
\end{abstract}

\begin{abstract}
In the Departments of Modern European Languages of Nigerian Universities, although translation forms an integral part of the academic programme, it is limited to French-English-French or German-English-German. Translating from French into the indigenous languages and vice versa is hardly conceived because of the country's colonial educational tradition. The aim of this paper is to translate the French voices into igbo, one of the three most important Nigerian languages, by raising the translation problems involved and proposing solutions.
\end{abstract}

\section{INTRODUCTION}

Des 26 universités conventionnelles nigérianes (16 fédérales et 10 régionales), il y en a à peine où les cours de traduction ne fassent partie intégrante du programme d'études françaises, qu'il s'agisse des institutions où le français tient le haut pavé dans le Département des langues modernes européennes ou bien de celles où la linguistique anglaise rivalise avec le français au sein du même Département des langues et de linguistique. Malheureusement, ces cours sont limités non seulement à l'orientation français-anglais-français (Okiwelu 1991) ou anglais-allemand-anglais mais encore, et dans la majorité des cas, à l'activité pratique: restitution pure et simple d'un texte écrit dans une autre langue. La stylistique comparée (Vinay et Darbelnet 1977) du français et de la langue maternelle de l'étudiant ne se conçoit jamais comme un objet méritant l'attention dans une classe de traduction. La négligence de la langue maternelle dans la traduction impliquant une langue étrangère est due aux habitudes longtemps acquises: respect pour la tradition du système éducatif colonial qui relègue les langues africaines au second plan et qui les réduit au statut de dialectes, de patois, « inaptes à véhiculer les idées scientifiques », refus à toute tentative visant à modifier le système dans un pays multilingue qui se trouve toujours dans l'impossibilité de se débarrasser de l'anglais comme langue officielle, d'adopter une langue indigène comme langue nationale. Cette négligence vient aussi des problèmes de sous-développement: ceux d'impression, de publication et de diffusion des matières rédigées sur les langues du pays. Pourtant, et contrairement à ce qu'en disent les pédagogues behavioristes, tels André Reboulet (1971), Denis Girard (1972), nous estimons en effet que l'apprentissage d'une langue étrangère comme c'est le cas du français au Nigeria aussi bien que l'acquisition d'un bilinguisme authentique seront largement facilités et garantis si l'étudiant maittrise déjà les structures linguistiques de sa langue maternelle. D’autre part, et en dehors du Nigéria, la domination des écrits en traduction par les langues indo-européennes a assez récemment retenu l'attention de Judy Wakabayashi (1991 : 415). Dans cet article célèbre, elle a qualifié, à juste titre, d'« eurocentrique » ce parti pris de la part des spécialistes en traduction. Et plus intéressant encore, elle a démontré, en s'appuyant sur l'exemple anglais-japonais, que la traduction entre deux langues qui sont géographiquement, culturellement, politiquement et socialement éloignées, est plus problématique que celle qui opère avec des langues de la même famille. C'est précisément le cas du français, langue indo-européenne et de l'igbo, langue de la famille kwa issue du Niger-Congo (Hansford et Bon Stanford 1976), laquelle est parlée par quelque 14 millions d'habitants qui peuplent essentiellement l'est du Nigeria (États d'Abia, d'Anambra, d'Enugu, d'Imo et, légèrement, l'État de Rivers) et partiellement l'État de Delta au sud-ouest du pays.

Notre propos est donc d'exposer les différences de structures pouvant gêner la traduction des voix françaises en igbo, puis de proposer des solutions. Nous espérons en définitive que cette comparaison permettra à l'étudiant nigérian et surtout igbophone de mieux comprendre et de mieux apprendre le français langue étrangère aussi bien que l'igbo langue maternelle dont il maîtrise d'ailleurs l'écrit. Nous 
espérons qu'elle lui servira également d'inspiration dans la conjoncture actuelle où la plupart des Nigérians qui ont une licence de français et qui désirent pousser leurs études plus loin optent le plus souvent pour la traduction en ce sens qu'elle leur ouvre une belle voie au métier de traducteur professionnel qu'ils considèrent de loin plus rentable et plus satisfaisant que l'enseignement. De même que pour le traducteur-chercheur, ce travail d'investigation risque heureusement de lui faire tomber sur quelque chose de nouveau et d'inattendu en traduction. Avant d'aborder les deux points qui viennent d'être annoncés, il conviendra de savoir ce que sont les voix françaises.

Le problème fondamental que pose la traduction des voix françaises est celui de la définition. Tout d'abord, combien de voix y a-t-il en français? Cette question sur laquelle les linguistes sont partagés est étroitement liée à celle de la définition proprement dite.

La grammaire traditionnelle limite le nombre de voix à deux seulement, à savoir l'actif et le passif, intégrant ainsi les verbes pronominaux et intransitifs à l'intérieur de la voix active, c'est-à-dire toute forme verbale autre que le couple être + participe passé issu des verbes transitifs directs. Dépassant ce cadre étroit de la vision diathétique (Tesnière 1959), le Dictionnaire de linguistique (1973: 512) reconnaît une troisième voix en français, voix moyenne, prise de position qui est justifiée par sa définition des voix en question. Ainsi, il y a la voix active «lorsque le sujet du verbe est l'agent de l'action qui s'exerce sur l'objet », la voix passive «lorsque le sujet de la phrase est [...] l'objet d'un verbe actif dans une phrase sousjacente", et enfin, la voix moyenne "si le sujet de la phrase est en même temps l'objet de l'action indiquée par le verbe (que ce sujet soit ou non l'agent de l'action)» (Dubois et al. 1973: 512). L'existence de la voix moyenne et la position intermédiaire qu'elle occupe par rapport à l'actif et au passif sont bien confirmées par Émile Benveniste (1966: 172) dans une définition encore plus précise: «Dans le moyen [...], le sujet est centre en même temps qu'acteur du procès; il accomplit quelque chose qui s'accomplit en lui. »

Tout en reconnaissant ces voix, Coste et al. $(1976: 241)$ en dégagent jusqu'à quatre en ajoutant à cette liste la voix attributive qu'ils définissent comme relevant de «l'état général d'un objet». Nous retiendrons cette définition, celle d'état, à cette différence qu'au lieu d'accorder la primauté à l'actance, nous mettrons l'accent sur les actants. Ainsi, dans une phrase attributive, l'actant 1 est le sujet qui est dans un état, sujet étatif ou simplement l'étatif, à qui on attribue quelque chose, une qualité, un métier, un phénomène, etc., représenté par l'actant 2 qui peut être adjectif participe ou nom/substantif. L'actant 2 joue le rôle attributif et constitue donc l'attribut. En définitive, la voix est active lorsque le sujet de l'énoncé est l'agent du procès indiqué par le verbe, passive lorsqu'il est patient, moyenne lorsqu'il est les deux à la fois, et attributive quand le sujet reçoit un attribut. Ces quatre rôles du sujet correspondent formellement et respectivement aux verbes transitifs, puis aux transitifs dont la vision actantielle est renversée (être $+p p)$, aux verbes pronominaux et intransitifs, et finalement, aux verbes de être. S'il est convenu que la voix est, comme la définissent globalement Coste et al. (1976:241), un actant, rappelons-le, pouvant être sujet, complément d'objet, les deux en même temps ou bien attribut, il sied maintenant de poser les problèmes qui se manifestent au cours de la traduction des voix françaises en igbo. Pour notre traduction, tous les tons igbo seront notés: / ton haut; \ ton bas; _ ton abaissé (downstep), sauf ce premier. La variante utilisée ici est l'igbo d'ntcha.

\section{LES PROBLÈMES LINGUISTIQUES}

Il s'agit de divergences entre les deux langues. Elles sont de cinq types: syntaxique, morphémique, lexical, morpho-sémantique et sémantique.

\section{La divergence syntaxique}

Bien qu'il existe également la voix active en igbo, la correspondance syntaxique (sujet + verbe + complément d'objet) entre les deux langues est interrompue d'abord au niveau de l'actif transitif direct lorsque le complément d'objet direct est pronominalisé en français, ce qui donne la séquence $\mathrm{S}+\mathrm{COD}+$ $\mathrm{V}$ en français et $\mathrm{S}+\mathrm{V}+\mathrm{COD}$ en igbo. Pour la traduction de l'actif transitif indirect, le même problème. L'igbo traduit les deux variantes syntaxiques françaises $\mathrm{S}+\mathrm{V}+\mathrm{COI}$ et $\mathrm{S}+\mathrm{COI}+\mathrm{V}$ par une seule variante $\mathrm{S}+\mathrm{V}+\mathrm{COI}$, le bouleversement de la correspondance étant dû encore à la pronominalisation du COI en français. Dans la traduction de la voix active à double transitivité, la correspondance syntaxique ne s'opère que lorsque le COD nominal est postposé au COI nominal en français. Dans les cinq autres cas, il y a une divergence structurale, laquelle est due à l'instabilité syntaxique des actants français. La même raison explique les cas de non-correspondance dans l'actif transitif direct et l'actif transitif indirect. Autrement dit, dans les trois parcelles actives: directe, indirecte et doublement transitive, le français a deux variantes combinatoires pour chacune des deux premières et jusqu'à six pour cette dernière, tandis que l'igbo a toujours une seule variante dans chaque cas. Ce que nous venons de dire est résumé ci-dessous, à cette 
différence que, pour des raisons de clarté, les termes sujet, complément d'objet direct et complément d'objet indirect seront remplacés respectivement par agent $(\mathrm{A})$, patient $1\left(\mathrm{P}^{1}\right)$ et patient $2\left(\mathrm{P}^{2}\right), \mathrm{P}$ étant patient en général.

1. La voix active transitive directe (2 variantes se traduisent par 1)

Marie balaie la maison:

Màrià nà àzà $n$

$$
\mathrm{A}+\mathrm{V}+\mathrm{P}^{1}
$$

$\mathrm{A}+\mathrm{V}+\mathrm{P}^{1}$

Marie la balaie:

$$
\mathrm{A}+\mathrm{P}^{1}+\mathrm{V}
$$

Màrià nà àzà ya

$\mathrm{A}+\mathrm{V}+\mathrm{P}^{1}$

2. La voix active transitive indirecte ( 2 variantes se traduisent par 1 )

Marie répond à Pierre:

$$
\mathrm{A}+\mathrm{V}+\mathrm{P}^{2} \quad \mathrm{~A}+\mathrm{V}+\mathrm{P}^{2}
$$

Màrià nà àza Pità

Marie lui répond:

$$
\mathrm{A}+\mathrm{P}^{2}+\mathrm{V}
$$

Màrià nà àza $y_{-}$

$$
\mathrm{A}+\mathrm{V}+\mathrm{P}^{2}
$$

3. La voix active doublement transitive (6 variantes se traduisent par 1 )

On traduit quand même neuf phrases pour la clarté:

Pierre donne de l'argent à Marie: Pità nà ènye Màrià eg

$$
\mathrm{A}+\mathrm{V}+\mathrm{P}^{1}+\mathrm{P}^{2}: \quad \mathrm{A}+\mathrm{V}+\mathrm{P}^{2}+\mathrm{P}^{1}
$$

Pierre lui donne de l'argent:

$$
\mathrm{A}+\mathrm{P}^{2}+\mathrm{V}+\mathrm{P}^{1} \text { : }
$$

Pità nà ènye $y_{-}$eg_

Pierre donne à Marie de l'argent:

$$
\mathrm{A}+\mathrm{V}+\mathrm{P}^{2}+\mathrm{P}^{1} \text { : }
$$

$$
\mathrm{A}+\mathrm{V}+\mathrm{P}^{2}+\mathrm{P}^{1}
$$

Pità nà ènye Màrià eg_

Pierre en donne à Marie :

$$
\mathrm{A}+\mathrm{P}^{1}+\mathrm{V}+\mathrm{P}^{2}
$$

$\mathrm{A}+\mathrm{V}+\mathrm{P}^{2}+\mathrm{P}^{1}$

Pità nà ènye Màrià fd

$\mathrm{A}+\mathrm{V}+\mathrm{P}^{2}+\mathrm{P}^{1}$

Pierre lui en donne:

$$
\mathrm{A}+\mathrm{P}^{2}+\mathrm{P}^{1}+\mathrm{V} \text { : }
$$

Pità nà ènye $y_{-} f d$

$\mathrm{A}+\mathrm{V}+\mathrm{P}^{2}+\mathrm{P}^{1}$

Pierre nous donne de l'argent:

$$
\mathrm{A}+\mathrm{P}^{2}+\mathrm{V}+\mathrm{P}^{1} \text { : }
$$

Pità nà ènye_nyi eg_

$\mathrm{A}+\mathrm{V}+\mathrm{P}^{2}+\mathrm{P}^{1}$

Pierre nous en donne:

$$
\mathrm{A}+\mathrm{P}^{2}+\mathrm{P}^{1}+\mathrm{V}
$$

Pità nà ènye_nyi fd

$\mathrm{A}+\mathrm{V}+\mathrm{P}^{2}+\mathrm{P}^{1}$

Pierre donne l'argent à Marie:

$$
\mathrm{A}+\mathrm{V}+\mathrm{P}^{1}+\mathrm{P}^{2} \text { : }
$$

Pità nà ènye Màrià ego af

Pierre le lui donne:

$$
\mathrm{A}+\mathrm{P}^{1}+\mathrm{P}^{2}+\mathrm{V}
$$

$\mathrm{A}+\mathrm{V}+\mathrm{P}^{2}+\mathrm{P}^{1}$

Pità nà ènye _ya

$\mathrm{A}+\mathrm{V}+\mathrm{P}^{2}+\mathrm{P}^{1}$

Ces différences expliquent l'impossibilité de traduire mot à mot dans la majorité des cas.

Pour la traduction de la voix moyenne, une différence similaire se manifeste entre les deux langues. Premièrement, au niveau de la voix moyenne réfléchie, ce que le français exprime par la séquence $\mathrm{A}+\mathrm{P}^{1}$ $+\mathrm{V}$, l'igbo traduit par $\mathrm{A}+\mathrm{V}+\mathrm{P}^{1}$. Il en va de même pour le réciproque direct et indirect où le patient est antéposé au verbe en français mais postposé en igbo, soit $A^{1}+A^{2}+P^{1}+V: A^{1}+A^{2}+V+P^{1}$ et $A^{1}+A^{2}$ $+P^{2}+V: A^{1}+A^{2}+V+P^{2}$. En ce qui concerne le réciproque issu des verbes doublement transitifs, le français a deux variantes combinatoires qui se traduisent par une seule en igbo $\left(\mathrm{A}^{1}+\mathrm{A}^{2}+\mathrm{P}^{2}+\mathrm{V}+\mathrm{P}^{1}\right.$ et $\left.\mathrm{A}^{1}+\mathrm{A}^{2}+\mathrm{P}^{2}+\mathrm{P}^{1}+\mathrm{V}: \mathrm{A}^{1}+\mathrm{A}^{2}+\mathrm{V}+\mathrm{P}^{2}+\mathrm{P}^{1}\right)$, la divergence syntaxique étant due non seulement à la différence spatiale fondamentale des deux langues mais aussi à la pronominalisation du $\mathrm{P}^{1}$ en français. Pour la voix moyenne des verbes intransitifs, on a une correspondance structurale partielle $(A / P+V)$, mais la même phrase peut se traduire également par une séquence de la voix active, soit: $A / P+V: A+V$ $+\mathrm{P}^{1}$, tandis que dans les autres cas, c'est toujours la séquence $\mathrm{A}+\mathrm{V}+\mathrm{P}^{1}$ qui s'impose en igbo. Résumons l'ensemble de ce qui vient d'être dit:

1. La voix moyenne réfléchie
Marie se lave:
Màrià nà àsa_ru

$$
\mathrm{A}+\mathrm{P}^{1}+\mathrm{V}
$$$$
\mathrm{A}+\mathrm{V}+\mathrm{P}^{1}
$$

2. La voix moyenne réciproque directe

Pierre et Marie se regardent:

$$
\mathrm{A}^{1}+\mathrm{A}^{2}+\mathrm{P}^{1}+\mathrm{V}
$$

Pità nà Màrià nà ène_nwe-fa $\mathrm{A}^{1}+\mathrm{A}^{2}+\mathrm{V}+\mathrm{P}^{1}$ 
3. La voix moyenne réciproque indirecte

Pierre et Marie se parlent:

$\mathrm{A}^{1}+\mathrm{A}^{2}+\mathrm{P}^{2}+\mathrm{V}$

Pità nà Màrià nà àgwa_nwe-fa

$\mathrm{A}^{1}+\mathrm{A}^{2}+\mathrm{V}+\mathrm{P}^{2}$

4. La voix moyenne réciproque des verbes doublement transitifs

Pierre et Marie se sont construit des maisons:

Pità nà Màrià ll ònwe-fa $n$

$\mathrm{A}^{1}+\mathrm{A}^{2}+\mathrm{P}^{2}+\mathrm{V}+\mathrm{P}^{1}$

Pierre et Marie s'en sont construit

$\mathrm{A}^{1}+\mathrm{A}^{2}+\mathrm{V}+\mathrm{P}^{2}+\mathrm{P}^{1}$

$\mathrm{A}^{1}+\mathrm{A}^{2}+\mathrm{P}^{2}+\mathrm{P}^{1}+\mathrm{V}$

Pità nà Màrià ll ònwe-fa_fodu

$\mathrm{A}^{1}+\mathrm{A}^{2}+\mathrm{V}+\mathrm{P}^{2}+\mathrm{P}^{1}$

5. La voix moyenne des verbes intransitifs

Le rocher bouge:

Òkwut_nà èkwerùbe ou Òkwut_nà èkwerùbe ekwerùbe $\mathrm{A} / \mathrm{P}+\mathrm{V}$ $\mathrm{A} / \mathrm{P}+\mathrm{V}$ ou $\mathrm{A}+\mathrm{V}+\mathrm{P}^{1}$

Pierre dort: Pità nà àlar ul_ $\mathrm{A} / \mathrm{P}+\mathrm{V}$ $\mathrm{A}+\mathrm{V}+\mathrm{P}^{1}$

Ce serait donc une erreur de traduction que de suivre toujours mot à mot en igbo la séquence syntaxique française.

La divergence atteint son niveau le plus haut avec la traduction de la voix passive en ceci que le passif n'existe pas en igbo. Ce fait est attesté par la syntaxe opposée des deux langues. Ce que le français exprime par la structure $\mathrm{P}+\mathrm{V}+\mathrm{A}$, l'igbo traduit obligatoirement par le détour actif:

Le lièvre est poursuivi par le chasseur

Dint_nà àch ulilì

$\mathrm{P}+\mathrm{V}+\mathrm{A}$

$\mathrm{A}+\mathrm{V}+\mathrm{P}$

Mot à mot: chasseur en train poursuivant lièvre.

L'inversion de l'ordre $\mathrm{A}+\mathrm{V}+\mathrm{P}$ en $\mathrm{P}+\mathrm{V}+\mathrm{A}$ est impossible en igbo. Même dans la traduction suivante où on s'acharne à opérer bon gré mal gré la transformation passive:

Ulili, dint_nà àchu y_

Mot à mot: Lièvre chasseur en train poursuivant le =

Le lièvre, le chasseur le poursuit

ou Ülilì, bù dint_nà àch $y_{-}$

Mot à mot: lièvre, ce est chasseur en train le poursuivant le $=$

Le lièvre, c'est le chasseur qui le poursuit.

Le placement du patient en tête ne donne toujours rien de passif. Au bout du compte, la vraie séquence reste $\mathrm{A}+\mathrm{V}+\mathrm{P}$.

En résumé, bien que les deux langues partagent la séquence fondamentale de l'actif $\mathrm{A}+\mathrm{V}+\mathrm{P}$, puis celle de $\mathrm{A}+\mathrm{V}+\mathrm{P}^{2}+\mathrm{P}^{1}$, où le sujet est toujours agent, la correspondance syntaxique est loin d'être totale dans l'ensemble, en raison du manque de stabilité spatiale des actants français déjà signalé. Sauf pour le cas en igbo (noté plus haut, "voix moyenne des verbes intransitifs"), la même raison rend compte de la divergence structurale au niveau de la voix moyenne. Dans la traduction du passif, la divergence syntaxique devient totale au point même de rendre la traduction impossible sinon par une déviation syntaxique. Le schéma ci-après fait ressortir les divergences en question:

a) la voix active transitive directe: $\quad \mathrm{A}+\mathrm{V}+\mathrm{P}^{1}$

b) la voix active transitive indirecte: $\begin{array}{ll}\mathrm{A}+\mathrm{P}^{1}+\mathrm{V} \\ \mathrm{A}+\mathrm{V}+\mathrm{P}^{2}\end{array}$

$$
\mathrm{A}+\mathrm{V}+\mathrm{P}^{1}
$$

$\mathrm{A}+\mathrm{V}+\mathrm{P}^{2}$

$$
\mathrm{A}+\mathrm{P}^{2}+\mathrm{V}
$$

$\mathrm{A}+\mathrm{V}+\mathrm{P}^{1}+\mathrm{P}^{2}$

$\mathrm{A}+\mathrm{P}^{2}+\mathrm{V}+\mathrm{P}^{1}$

$\mathrm{A}+\mathrm{V}+\mathrm{P}^{2}+\mathrm{P}^{1}$

$\mathrm{A}+\mathrm{V}+\mathrm{P}^{2}+\mathrm{P}^{1}$

$\mathrm{A}+\mathrm{P}^{1}+\mathrm{V}+\mathrm{P}^{2}$

$\mathrm{A}+\mathrm{P}^{2}+\mathrm{P}^{1}+\mathrm{V}$

$\mathrm{A}+\mathrm{P}^{1}+\mathrm{P}^{2}+\mathrm{V}$ 
d) la voix moyenne réfléchie:

e) la voix moyenne réciproque directe:

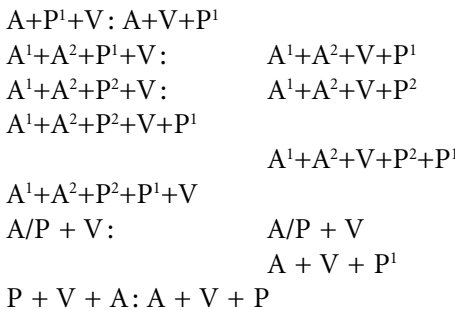

$\mathrm{P}+\mathrm{V}+\mathrm{A}: \mathrm{A}+\mathrm{V}+\mathrm{P}$

i) la voix passive:

Ces divergences constituent donc une mise en garde contre la traduction littérale.

\section{La divergence morphémique}

Il s'agit de différences au niveau des morphèmes, le morphème étant pris au sens distributionnel du terme: «Le plus petit élément significatif individualisé dans un énoncé» (Dubois et al. 1973). On constate d'abord que dans l'expression de la voix réfléchie, le français se sert d'un morphème pronominal pour marquer l'actant $\mathrm{n}^{\circ} 2$ tandis que l'igbo le traduit très souvent par un morphème nominal. Reprenons un exemple déjà cité:

\section{Marie se lave: Màrià nà àsa_ru \\ pronom nom}

De même que pour exprimer la voix moyenne réciproque, la valeur réciproque en français est toujours marquée par un morphème pronominal mais peut être également un morphème suffixal (-tali) en igbo:

$\begin{array}{ll}\begin{array}{l}\text { Pierre et Marie śécrivent: } \\ \text { morphème pronominal }\end{array} & \text { Pità nà Màrià nà édetali akwu kwo } \\ \text { morphème lexèmique morphème suffixal }\end{array}$

L'erreur de traduction consisterait à chercher un pronom en igbo pour traduire celui du français dans cdeux derniers exemples, ce qui donnerait les variantes suivantes:

Màrià nà àsa_nwe-ya aru

Mot à mot: Marie en train lavant elle-même corps

Marie se lave elle-même

Pità nà Màrià nà èdeli_nwe-fa akwkw

littéralement: Pierre et Marie écrivent à/pour/par eux-mêmes.

Ces rendements sont grammaticalement corrects mais constituent un contresens pour Marie se lave et restent ambigus pour Pierre et Marie s'écrivent par rapport au message de la langue source. Cela veut dire que la traduction de pronoms réfléchi et réciproque français par le même pronom en igbo, quoique possible (cf. cette fille est belle et Pierre et Marie se parlent), ne marche pas dans tous les cas.

\section{La divergence lexicale}

Pour exprimer les voix, l'igbo n'utilise ni le même type ni le même nombre d'éléments lexicaux que le français dans tous les cas. Dans la traduction de la voix attributive, l'idée d'attribut que le français exprime par un adjectif, l'igbo la traduit par un substantif. Par exemple:

Cette fille est belle: $\quad$ Nwagbogh_à dì $\mathrm{mm}_{-}$

adjectif substantif

Mot à mot: Fille ce est beauté

Bien que l'adjectif oma (beau) existe, les adjectifs étant très rares d'ailleurs en igbo, ce sera une erreur de traduction parce qu'agrammatical que de dire: «Nwagbogh_à dì oma.» En igbo, l'expression de qualification en général est réalisée par la juxtaposition de noms et celle d'attribution, par la postposition d'un nom au verbe être, comme ici.

Quant au nombre d'éléments, la traduction de la voix active, par exemple, ne donne pas toujours la même quantité lexicale dans les deux langues. L'actif transitif direct qui se réalise de façon absolue, c'està-dire sans patient exprimé, se traduit, au contraire, par un patient: 
$\begin{array}{ll}\text { Marie chante : } & \text { Màrià nà èkwe _kwe } \\ 1 \text { agent } & 1 \text { agent }\end{array}$

Mot à mot: Marie en train chantant chanson

Il en est ainsi puisque le verbe actif igbo exige presque toujours un complément nominal. La même nature de différence lexicale et la même explication valent pour la traduction de l'actif à double transitivité. Ce que le français exprime par deux actants seuls, l'igbo traduit obligatoirement par trois:

Pierre écrit à Marie: $\quad$ Pità nà èdeli Màrià akwukwo

$\begin{array}{lllll}1 & 2 & 1 & 2 & 3\end{array}$

Il y a très souvent en igbo un nom cognat dérivé du verbe et qui complète ledit verbe. D'où l'actant supplémentaire par rapport au français. Négliger cet élément nominal en traduction aboutira à une phrase inachevée.

Pour la bonne traduction des voix françaises en igbo, il est donc impératif de savoir la nature aussi bien que le nombre d'éléments lexicaux qui s'imposent en igbo pour une expression équivalente. Seul un bon niveau de bilinguisme sera une garantie contre une erreur de traduction.

\section{La divergence morpho-sémantique}

Y a-t-il dans le sens des morphèmes diathétiques des deux langues quelque chose qui gêne la traduction? Autrement dit, le rapport qui unit le signifiant au signifié, pour reprendre la terminologie saussurienne, est-il le même dans la langue cible que celui qui les unit dans la langue source? Étudions cette question d'abord sur le plan de la voix moyenne réfléchie. Si dans la traduction:

Pierre se peigne: Pità nà àracha is_,

on remarque, bien qu'il s'agisse globalement de la même idée de réfléchi, que le lien sémantique qui unit peigne à l'actant 2 (se) est plus étroit et plus fort que celui qui s'instaure entre nà àracha et l'actant 2 (is_ $=$ tête). Ce que le français considère comme une action faite et subie par la totalité du sujet, l'igbo le voit comme une action faite par le sujet mais subie seulement par une partie de cette totalité. Toute tentative visant à chercher ici une correspondance totale entre le morphème et le sens dans les deux langues et donc d'introduire un élément pronominal en igbo dans l'exemple ci-dessus aboutirait soit à une phrase agrammaticale, incomplète, telle que: Pità nà aracha_nwe-ya, soit à un contresens comme Pità nà àracha _nwe-ya isi (cf. plus haut Marie se lave et Pierre et Marie s'écrivent). Dans ce cas, on n'a qu'à se contenter d'une sorte de parallélisme sémantique. De plus, dans le moyen réciproque, le lien existant entre le morphème et sa valeur sémantique dans chacune des langues n'est pas toujours d'une égale importance. C'est ainsi qu'en français un seul morphème, se, renvoie simultanément à deux valeurs diathétiques, à l'idée de patient d'une part, et à celle de réciprocité d'autre part. Par contre, en igbo, à chaque signifiant correspond un signifié, comme l'atteste cet exemple:

Pierre et Marie s'embrassent: patient + réciprocité
Pità nà Màrià nà ètilitali obì réciprocité patient

Il s'ensuit que le traducteur non igbophone, à force de rechercher une correspondance morphosémantique, risquerait de traduire la même phrase ainsi:

Pità nà Màrià nà ètili_nwe-fa

Mot à mot: Pierre et Marie en train jouant pour eux-mêmes (un tam-tam, par exemple), rendement qui constitue un contresens. Ou bien on retient la première traduction avec la marque réciproque -tali, ou bien on a, dans ce cas:

\section{Pità nà Màrià nà ètili obì}

où l'idée de réciprocité est marquée implicitement. Mais il faut le complément nominal obi qui fait partie du verbe et qui en complète le sens. C'est là encore la différence entre le français et l'igbo. La partie du corps par où l'embrassement se fait (obi = poitrine) est marquée explicitement en igbo mais sousentendue en français. La façon dont chaque langue répartit les phénomènes sémantiques à leurs signifiants est un facteur à ne pas négliger en traduction. 


\section{La divergence sémantique}

Les problèmes linguistiques de la traduction atteignent leur niveau plus haut lorsqu'il y a une divergence sémantique, c'est-à-dire quand le signifié de LS n'existe pas en LC que LC traduit le même message par un signifié différent. C'est ce qui se produit d'abord pour la traduction d'une partie de la voix attributive, laquelle se rend en igbo par la voix moyenne:

$$
\begin{array}{ll}
\text { Le pot est cassé: } & \text { Itè ètiwago } \\
\mathrm{E}+\text { aux. }+ \text { Att.: } & \mathrm{A} / \mathrm{P}+\mathrm{V} \\
\text { (voix attributive) } & \text { (voix moyenne) }
\end{array}
$$

Mot à mot: Pot casser + marque d'accompli -go

$$
\begin{array}{ll}
\text { Ce livre est très lu: } & \text { A nà àg akwkw à } r n n_{-} \\
\begin{array}{l}
\mathrm{E}+\text { aux. + Att.: } \\
\text { (voix attributive) }
\end{array} & \mathrm{A}+\mathrm{VTD}+\mathrm{P} \\
\text { (voix active) }
\end{array}
$$

Mot à mot: On est en train lisant livre ce beaucoup

La raison d'ensemble est que la langue igbo ne dispose ni de verbe être en tant qu'auxiliaire ni de participe passé pour jouer le rôle d'attribut, car c'est le point de départ de la voix attributive. De plus, la deuxième phrase est l'équivalent d'une véritable phrase passive, le sujet étant complètement patient. L'igbo ne peut donc que la traduire par l'actif. Pour la première, le sujet est à la fois agent et patient dans les deux langues, en ce sens que le pot peut se casser tout seul ou bien être cassé par quelqu'un ou quelque chose. Pourtant, la différence sémantique vient de ce que le français accorde ici l'importance à l'attribution de l'action au sujet, tandis que l'igbo voit le même phénomène comme une action à la fois agie et subie par le sujet.

Pour la traduction de la voix moyenne, il existe également une divergence sémantique. Soit cet exemple à construction intransitive:

$\begin{array}{ll}\text { Pierre dort: } & \text { Pità nà àlaru ul } \\ \mathrm{A} / \mathrm{P}+\mathrm{V}: & \mathrm{A}+\mathrm{VTD}+\mathrm{P} \\ \text { (voix moyenne) }: & \text { (voix active) }\end{array}$

Ce que le français considère comme un phénomène agi et subi est en igbo une action par excellence. De même que la traduction de la voix moyenne suivante ne se fera que par le biais de la voix active:

\section{Ce fruit se mange cru: A nà àta mkpulu-osisi à na nd}

Mot à mot: On en train mangeant fruit ce dans cru

La raison en est que l'agent de la phrase source est indéterminé et que le sujet est moins agent que patient. Or, la traduction de la voix moyenne par la même voix en igbo n'est opératoire que si les possibilités du sujet à poser comme agent ou comme patient sont à valeur égale. Par contre, dans tous ces exemples, le sujet est presque entièrement patient. En réalité, c'est une vraie phrase passive camouflée sous forme de voix moyenne. Elle est dérivée de la phrase passive sous-jacente: "Ce fruit est mangé cru", laquelle est dérivée à son tour de la phrase active sous-jacente: "On mange ce fruit cru», la voix active étant le point de départ de toute construction passive. Il s'ensuit que la divergence sémantique arrive au sommet quand il s'agit de traduire la voix passive. Soit:

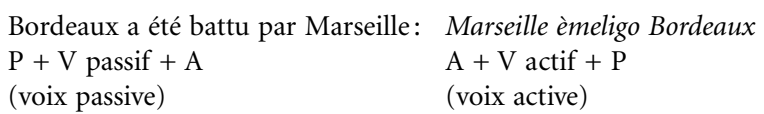

La divergence sémantique vient de l'impossibilité patient à poser comme sujet en igbo. D'où aussi l'impossibilité du verbe igbo à être renversé dans la vision actantielle. La différence sémantique est un élément à ne pas perdre de vue dans une activité traduisante, parce qu'elle est la plus poussée de toutes les problématiques linguistiques et que la sémantique est le dernier recours pour trancher toute divergence d'ordre formel en traduction.

Comment résoudre alors les problèmes divers qui ont été soulevés au cours de ce chapitre? C'est ce qui nous reste à chercher. 


\section{SOLUTIONS}

Les solutions sont étroitement liées d'abord aux facteurs de production des données linguistiques exprimant la voix dans les deux langues, puis à ceux de transfert du message. Dans le premier cas, il s'agit de phénomènes sémantiques et dans le deuxième, il est question des techniques de traduction. Ainsi, quand la voix est créée dans les deux langues par le même phénomène sémantique, on traduit soit littéralement soit par ce que Vinay et Darbelnet (1977: 50) appellent «transposition ", à cette différence près que la transposition peut s'opérer non seulement au niveau des parties du discours, opération que nous avons baptisée transposition formelle, quand il y en a. Elle peut s'opérer également sur le plan syntaxique (transposition structurale). Lorsque l'existence des éléments linguistiques est conditionnée par des critères sémantiques différents, on traduit par l'adaptation (Vinay et Darbelnet 1977: 52), la différence sémantique s'expliquant par des phénomènes extra-linguistiques tels que la culture et la vision du monde (Mounin 1962). Nous expliciterons le mécanisme de la traduction au fur et à mesure. Seront utilisés en plus les signes et sigles suivants: $\rightarrow$ (donne), // (se transpose en), VTD (verbe transitif direct), VTI (verbe transitif indirect), VDT (verbe doublement transitif), $\mathrm{PN}^{1}$ (patient nominal direct), $\mathrm{PN}^{2}$ (patient nominal indirect), $\mathrm{P} \varnothing$ (patient zéro), $\mathrm{Ppr}^{1}$ (patient pronominal direct), $\mathrm{Ppr}^{2}$ (patient pronominal indirect), $\mathrm{PprR}$ (patient pronominal réfléchi), $\mathrm{VSR}^{\mathrm{c}}$ (verbe à suffixe réciproque), $\mathrm{PprRc}$ (patient pronominal réciproque), Att. (attribut), Att.P (attribut participial), Ppr.S (patient pronominal subjectif), E (étatif), VP (verbe passif), VA (verbe actif), Att.adj. (attribut adjectival), Att.N (attribut nominal).

\section{La traduction de la voix active}

La voix active crée en LS et LC par l'activité du sujet, le sujet étant agent, on traduit soit littéralement soit par transposition.

1) Traduction littérale

a) de l'actif transitif direct

$\rightarrow \mathrm{A}+\mathrm{VTD}+\mathrm{PN}^{1}$ :

$\mathrm{A}+\mathrm{VTD}+\mathrm{PN}^{1}$

$\rightarrow$ Marie balaie la maison:

Màrià nà àzà $n$

b) de l'actif transitif indirect

$\rightarrow \mathrm{A}+\mathrm{VTI}+\mathrm{PN}^{2}$ :

$\mathrm{A}+\mathrm{VTI}+\mathrm{PN}^{2}$

$\rightarrow$ Marie répond à Pierre:

c) de l'actif doublement transitif

$\rightarrow \mathrm{A}+\mathrm{VDT}+\mathrm{PN}^{2}+\mathrm{PN}^{1}: \quad \mathrm{A}+\mathrm{VDT}+\mathrm{PN}^{2}+\mathrm{PN}^{1}$

$\rightarrow$ Pierre donne à Marie de l'argent: Pità nà ènye Màrià eg

d) de l'actif transitif absolu

$\rightarrow \mathrm{A}+\mathrm{VTD}+\mathrm{P} \varnothing:$

$\mathrm{A}+\mathrm{VTD}+\mathrm{P}^{1}$

$\rightarrow$ Pierre écrit:

Pità nà ède if

2) Traduction par transposition structurale

a) de l'actif transitif direct, $\mathrm{Ppr}^{1}+\mathrm{VTD} / / \mathrm{VTD}+\mathrm{Ppr}^{1}$

$\rightarrow \mathrm{A}+\mathrm{Ppr}^{1}+\mathrm{VTD}: \quad \mathrm{A}+\mathrm{VTD}+\mathrm{Ppr}^{1}$

$\rightarrow$ Marie la balaie: $\quad$ Màrià nà àzà ya

b) de l'actif transitif indirect, $\mathrm{Ppr}^{2}+\mathrm{VTI} / / \mathrm{VTI}+\mathrm{Ppr}^{2}$

$\rightarrow \mathrm{A}+\mathrm{Ppr}^{2}+\mathrm{VTI}: \quad \mathrm{A}+\mathrm{VTI}+\mathrm{Ppr}^{2}$

$\rightarrow$ Marie lui répond: $\quad$ Màrià nà àza $y_{-}$

c) de l'actif doublement transitif, $\mathrm{VDT}+\mathrm{PN}^{1}+\mathrm{PN}^{2}, \mathrm{Ppr}^{2}+\mathrm{VDT}+\mathrm{PN}^{1}, \mathrm{Ppr}^{1}+\mathrm{VDT}+\mathrm{PN}^{2}, \mathrm{Ppr}^{2}+\mathrm{Ppr}^{1}+\mathrm{VDT}$, $\mathrm{Ppr}^{1}+\mathrm{Ppr}^{2}+\mathrm{VDT} / / \mathrm{VDT}+\mathrm{P}^{2}+\mathrm{P}^{1}$, où $\mathrm{P}^{2}$ et $\mathrm{P}^{1}$ peuvent être nominal ou pronominal suivant le cas en LS.

c1) $\rightarrow \mathrm{A}+\mathrm{VDT}+\mathrm{PN}^{1}+\mathrm{PN}^{2}:$

$\mathrm{A}+\mathrm{VDT}+\mathrm{PN}^{2}+\mathrm{PN}^{1}$

$\rightarrow$ Pierre donne de l'argent à Marie :

Pità nà ènye Màrià eg

c2) $\rightarrow \mathrm{A}+\mathrm{Ppr}^{2}+\mathrm{VDT}+\mathrm{PN}^{1}$ :

$\mathrm{A}+\mathrm{VDT}+\mathrm{Ppr}^{2}+\mathrm{PN}^{1}$

$\rightarrow$ Pierre lui donne de l'argent:

Pità nà ènye $y_{-}$eg

c3) $\rightarrow \mathrm{A}+\mathrm{Ppr}^{1}+\mathrm{VDT}+\mathrm{PN}^{2}$ :

$\mathrm{A}+\mathrm{VDT}+\mathrm{PN}^{2}+\mathrm{Ppr}^{1}$

$\rightarrow$ Pierre en donne à Marie:

c4) $\rightarrow \mathrm{A}+\mathrm{Pp}^{2}+\mathrm{Ppr}^{1}+\mathrm{VDT}$ :

Pità nà ènye Màrià $f d$

$\rightarrow$ Pierre lui en donne:

$\mathrm{A}+\mathrm{VDT}+\mathrm{Ppr}^{2}+\mathrm{Ppr}^{1}$

Pità nà ènye ya $f d$

c5) $\rightarrow \mathrm{A}+\mathrm{Ppr}^{1}+\mathrm{Ppr}^{2}+\mathrm{VDT}$ :

$\mathrm{A}+\mathrm{VDT}+\mathrm{Ppr}^{2}+\mathrm{Ppr}^{1}$

$\rightarrow$ Pierre le lui donne:

Pità nà ènye _ya 
La traduction de la voix moyenne

La traduction de la voix moyenne soit par transposition soit par adaptation soit littéralement selon le sens du sujet en LS et en LC.

1) Réfléchie. Le sujet étant A et $\mathrm{P}$ en LS et en LC mais les deux patients étant de catégories et syntaxe différentes, on traduit:

a) soit par transposition structurale, $\mathrm{PprR}+\mathrm{V} / / \mathrm{V}+\mathrm{PprR}$

$\rightarrow \mathrm{A}+\mathrm{PprR}+\mathrm{V}: \quad \mathrm{A}+\mathrm{V}+\mathrm{PprR}$

$\rightarrow$ Marie se voit bien dans cette photo: Màrià nà àfu onwe-ya fuma nime foto à

b) soit par transposition structuro-formelle, $\mathrm{PprR}+\mathrm{V} / / \mathrm{V}+\mathrm{PNR}$

$\rightarrow \mathrm{A}+$ PprR + V: $\quad \mathrm{A}+\mathrm{V}+\mathrm{PNR}$

$\rightarrow$ Marie se lave: Màrià nà àsa ar

2) Réciproque traduction par transposition ou par adaptation

a) le réciproque traduit par transposition structuro-formelle, $\mathrm{PprRc}+\mathrm{V} / / \mathrm{VSRc}+\mathrm{PN}^{1}$

$\rightarrow \mathrm{A}^{1}+\mathrm{A}^{2}+\mathrm{PprRc}+\mathrm{V}: \quad \mathrm{A}^{1}+\mathrm{A}^{2}+\mathrm{VSRc}+\mathrm{PN}^{1}$

$\rightarrow \quad$ Pierre et Marie se : $\quad$ Pità nà Màrià nà àmatali_la

b) ou par transposition structurale, $\mathrm{PprRc}+\mathrm{V} / / \mathrm{V}+\mathrm{PprRc}$

$\rightarrow \mathrm{A}^{1}+\mathrm{A}^{2}+\mathrm{PprRc}+\mathrm{V}: \quad \mathrm{A}^{1}+\mathrm{A}^{2}+\mathrm{V}+\mathrm{PprRc}$

$\rightarrow \quad$ Pierre et Paul se battent: Pità nà Polù nà èti_nwe-fa

c) le réciproque traduit par adaptation (voix moyenne: voix active)

$\rightarrow \mathrm{A}^{1}+\mathrm{A}^{2}+\mathrm{PprRc}+\mathrm{V}: \quad \mathrm{A}^{1}+\mathrm{A}^{2}+\mathrm{VTD}+\mathrm{PN}^{1}$

$\rightarrow \quad$ Pierre et Paul se battent: Pità nà $P l$ nà àn $g$

Mot à mot: Pierre et Paul en train faisant combat

3) La voix moyenne au sens passif (traduction par adaptation voix moyenne: voix active)
( $\mathrm{A} / \mathrm{P}+\mathrm{P}+\mathrm{V}$ :
$\mathrm{A}+\mathrm{V}+\mathrm{P}^{1}$

a) (Ce fruit se mange cru: An nà àta mkpl-osisi à na nd

Mot à mot: On en train mangeant fruit ce dans cru

b) ( Le vin se vend bien cette année: A nà èle manya fuma $a r_{-} a ̀$

Mot à mot: On en train vendant vin bien année ce

4) La voix moyenne issue des verbes "essentiellement pronominaux»

Ce sont des pronominaux dont le pronom a une réalité subjective (Mounin 1962). On traduit:

a) soit littéralement, à cette différence près que l'élément pronominal est supprimé en LC.

$\rightarrow \mathrm{A}+\mathrm{PprS}+\mathrm{V}: \quad \mathrm{A} / \mathrm{P}+\mathrm{V}$

$\rightarrow$ Qu'est-ce qui ce passe?: O bù gini nà ème?

Mot à mot: est quoi en train arrivant

$\rightarrow \quad$ La réunion se déroule bien:

Nzùk nà àga fma

Mot à mot: Réunion en train allant bien

b) soit par adaptation (voix moyenne: voix active)
$\rightarrow \mathrm{A}+\mathrm{PprS}+\mathrm{V}$ :
$\mathrm{A}+\mathrm{VTD}+\mathrm{PN}^{1}$

$\rightarrow$ Cathie s'amuse:

Mot à mot: Cathie en train jouant jeu

5) La voix moyenne des verbes intransitifs - traduction:

a) soit littérale

$\rightarrow \mathrm{A} / \mathrm{P}+\mathrm{V}$ :

$\rightarrow$ Le rocher bouge:

Kàti nà ègwu egw_

Mot à mot: Rocher en train bougeant

$\rightarrow$ L'enfant grandit:

Mot à mot: Enfant en train grandissant

b) soit par adaptation (moyen: actif)

$\rightarrow \mathrm{A} / \mathrm{P}+\mathrm{V}$ :

$\mathrm{A} / \mathrm{P}+\mathrm{V}$

Okwut_nà èkwerùbe

$\rightarrow$ Pierre dort:

Mot à mot: Pierre en train dormant sommeil

$\rightarrow$ Le rocher bouge:

Nwatà nà àgbà

Mot à mot: Rocher en train bougeant bouger 


\section{La traduction de la voix attributive — traduction littérale ou par adaptation}

1) La voix attributive issue des verbes de être au sens plein — traduction littérale, par transposition ou par adaptation:

a) l'attribution d'un métier ou d'un objet de possession — se traduit littéralement:

$\rightarrow \mathrm{E}+\mathrm{V}+$ Att. : $\quad \mathrm{E}+\mathrm{V}+$ Att.

$\rightarrow$ Il est professeur: Obù onye-nkuzi

$\rightarrow$ Jacques a de l'argent: Jemisì nwè eg_

b) l'atribution d'une qualité physique ou morale _ se traduit:

b1) soit par transposition _ adjectif // nom

$\rightarrow \mathrm{E}+\mathrm{V}+$ Att. Adj.: $\quad \mathrm{E}+\mathrm{V}+$ Att.N

$\rightarrow$ Ada est belle: Ada dì $\mathrm{mm}_{-}$

Mot à mot: Ada est beauté

$\rightarrow$ L'âne est stupide: Obunnu-tàbà dì nzuzù

Mot à mot: Âne est stupidité

b2) soit par adaptation (attributif: actif)

$\rightarrow$ E + V + Att.: $\quad \mathrm{A}+\mathrm{VTD}+\mathrm{p}^{1}$

$\rightarrow$ Ada est belle: Ada mà $\mathrm{mm}_{-}$

Mot à mot: Ada embellit beauté

$\rightarrow \quad$ Il est stupide: $\quad$ nà èzuzù nzuzù

Mot à mot: Il en train faisant (la) bête bêtise

2) La voix attributive du participe attributif à lexème imperfectif - traduction par adaptation (attributif: actif)

$\rightarrow$ E + aux (être) + Att.P: $\quad \mathrm{A}+\mathrm{VTD}+\mathrm{P}^{1}$

$\rightarrow$ Ce livre est très lu: A nà àgu akwukwo à rinn_

Mot à mot: On en train lisant livre ce beaucoup

3) La voix attributive du participe à lexème perfectif _ traduction par adaptation (attributif: moyen)

$\rightarrow$ E + aux (être) + Att.P: $\quad$ A/P + V (-go)

$\rightarrow$ La tasse est cassée: Iko ètiwago

Mot à mot: Tasse cassant + marque d'accompli (-go)

La traduction de la voix passive - traduction par adaptation (passif: actif)

$\rightarrow \mathrm{P}+\mathrm{VP}+\mathrm{A}: \quad \mathrm{A}+\mathrm{VA}+\mathrm{P}^{1}$

$\rightarrow \quad$ Marie est aimée de Pierre: Pità fùlù Màrià na anya

Mot à mot: Pierre voit Marie aux yeux

\section{CONCLUSION}

Ce travail a montré qu'en dépit des divergences linguistiques qui existent entre les deux langues, les voix françaises peuvent se traduire en igbo. Ainsi, la traduction de la voix active, malgré l'instabilité des actants français en particulier, a été possible littéralement ou par transposition, grâce au sémantique que partagent les deux langues, le sujet étant toujours agent et l'objet toujours patient aussi bien en LS qu'en LC. Autrement dit, la voix active française se traduit par la voix active en igbo à cent pour cent, soit quatre fois sur dix littéralement six fois sur dix par la transposition. Pour la voix moyenne, la traduction du réfléchi et du réciproque par la transposition dans la grande majorité des cas (sept fois sur dix) a été rendu possible également par le sémantique _ activité plus passivité du sujet. Dans les autres cas, la voix moyenne se traduit au sein du même groupe soit littéralement quand le verbe admet un sujet à la fois agent et patient et qu'il y a une correspondance structuro-sémantique, soit par l'adaptation dans le cas contraire. En somme, la voix moyenne française se traduit par la voix moyenne en igbo dans la majorité des cas, soit six fois sur dix (2: 10 littéralement, 4:10 par transposition) et par adaptation quatre fois sur dix, la raison pour les cas d'adaptation étant que les verbes igbo exigent presque toujours un complément d'objet nominal. Quant à la voix attributive, elle se traduit mot à mot seulement lorsqu'on attribue un métier ou un objet de possession au sujet, soit $2: 10$. Pour les autres cas, elle se traduit soit par transposition au sein du même groupe $(2: 10)$ soit uniquement par adaptation $(6: 10)$. Quand on aborde la voix passive, on s'éloigne complètement de la traduction littérale au point même d'atteindre le degré d'intraduisibilité, la raison en étant que cette voix est inexistante en igbo. Persister à rechercher coûte que coûte une phrase passive en igbo ressemblerait à ce missionnaire de l'Amérique latine dont se sont bien moqués Taber et Nida (1971:3) d'avoir insisté «pour introduire une forme passive dans une langue indienne qui n'en avait pas ». Pourtant, il a été possible de franchir le seuil d'intraduisibilité et donc de rendre le même message par le détour actif, grâce au phénomène d'adaptation (Vinay et Darbelnet 1977: 52). 
En définitive, il s'avère que la seule voix qui n'est point en dispute en igbo est la voix active. Voilà pourquoi l'igbo, en dernier ressort, a tendance à recourir à cette voix presque chaque fois que la traduction mot à mot ou transposée échoue. Les exemples abondent, soit moyen : actif, attributif: actif. C'est que les deux langues n'ont pas la même façon d'opérer ni de voir la même expérience humaine dans bien des cas.

Cette divergence a une dimension culturelle, de même qu'elle est intimement liée à la conception différente que les deux communautés linguistiques ont du monde. Ainsi, pour la communauté igbo, le monde serait un univers clos, voire uni, où l'homme est toujours fait pour agir, où sa passivité est presque entièrement exclue, où le rapport agent-patient, celui de l'homme avec son semblable, resterait pour ainsi dire à sens unique, partant, stable. Par contre, pour la communauté française, le monde serait un univers large, voire instable, où existence et survivance ne seraient assurées que par un jeu oscillatoire et diplomatique entre les hommes, celui d'activité, de passivité, de juste milieu ou bien d'attribution, suivant l'occasion. Ces visions du monde antithétiques, d'un côté quasi univoque et, de l'autre, universalisante, rendent compte, au fond, des divergences linguistiques et éclairent par là même la traduction des voix françaises en igbo.

BENEDICT O. OKIWELU Université de Calabar, Calabar, Nigeria

\section{RÉFÉRENCES}

Benvéniste, Émile (1966): Problèmes de linguistique générale, 1, coll. «Tel», Paris, Gallimard.

Chevalier, J.-C. et al. (1964): Grammaire Larousse du français contemporain, Paris, Larousse.

Coste, D., J. Courtillon, V. Ferenzi, M. Martini-Baltar, et E. Papo (1976): Un niveau-seuil. Éducation et culture, Strasbourg, CREDIF.

Dubois, J., M. Giacomo, L. Guespin et al. (1973): Dictionnaire de linguistique, Paris, Larousse.

Girard, D. (1972) : Linguistique appliquée et didactique des langues, Paris, Armand Colin Longman.

HANsford, Keir et Samuel Bon STANFord (1976): Studies in Nigerian Languages, 5, Summer Institute of Linguistics, Accra, Ghana.

Mackey, William F. (1976): Bilinguisme et contacts des langues, Paris, Klincksieck.

Mounin, G. (1962): Les problèmes théoriques de la traduction, coll. "Tel », Paris, Gallimard.

OKiwelu, B. O. (1991): La traduction de l'aspect verbal français en igbo, Meta, 36 (2/3), p. 471-483.

Reboulet, A. (1971): Guide pédagogique pour le professeur de français langue étrangère, Paris, Hachette.

TABer, C. R. et E. A. NidA (1971): La traduction: théorie et méthode, London, New York, Stuttgart, Alliance biblique universelle.

Tesnière, L. (1959) : Éléments de syntaxe structurale, Paris, Klincksieck.

Vinay, J.-P. et J. Darbelnet (1977): Stylistique comparée du français et de l'anglais, Paris, Didier Érudition.

Wagner et Pichon (1962): Grammaire du français classique et moderne, Paris, Hachette.

WaKabayashi, Judy (1991): "Translation Between Unrelated Languages and Cultures, as Illustrated by Japanese-English Translation», Meta, 36 (2/3), p. 414-423. 